\title{
Avaliação em laboratório da atividade de voo de Rhodnius brethesi Matta, 1919, potencial vetor silvestre do Trypanosoma cruzi na Amazônia Brasileira. (Hemiptera: Reduviidae:Triatominae)
}

Laboratory analysis of the flight of Rhodnius brethesi Matta, 1919, potential wild vector of Trypanosoma cruzi in the Brazilian Amazon. (Hemiptera:Reduviidae:Triatominae)

Evaluación en laboratorio de la actividad de vuelo de Rhodnius brethesi Matta, 1919, potencial vector silvestre del Trypanosoma cruzi en la Amazonía Brasileña. (Hemiptera:Reduviidae:Triatominae)

\section{Dayse da Silva Rocha}

Laboratório Nacional e Internacional de Referência em Taxonomia de Triatomíneos, Instituto Oswaldo Cruz/MS, Rio de Janeiro, Rio de Janeiro, Brasil

\section{Claudia Solano}

Departamento de Ciências Biológicas, Faculdade de Ciências Farmacêuticas, Universidade Estadual Paulista, Araraquara, São Paulo, Brasil

\section{José Jurberg}

Laboratório Nacional e Internacional de Referência em Taxonomia de Triatomíneos, Instituto Oswaldo Cruz/MS, Rio de Janeiro, Rio de Janeiro, Brasil

\section{Vanda Cunha}

Laboratório Nacional e Internacional de Referência em Taxonomia de Triatomíneos, Instituto Oswaldo Cruz/MS, Rio de Janeiro, Rio de Janeiro, Brasil

\section{Cleber Galvão}

Laboratório Nacional e Internacional de Referência em Taxonomia de Triatomíneos, Instituto Oswaldo Cruz/MS, Rio de Janeiro, Rio de Janeiro, Brasil

\section{RESUMO}

A partir de observações realizadas em laboratório, foi avaliada a capacidade de voo de Rhodnius brethesi, correlacionando esta atividade a fatores como quantidade de sangue ingerida, gênero e período de jejum. Foram observados 27 machos e 38 fêmeas. O período médio de sobrevida foi de 17,8 e 22,3 dias para machos e fêmeas, respectivamente. A maioria dos insetos necessitov em média de 13 dias para iniciar o voo.

Palavras-chave: Rhodnius; Doença de Chagas; Triatominae; Insetos Vetores; Comportamento Animal; Voo Animal.

\section{INTRODUÇÃO}

Os triatomíneos constituem uma subfamília de hemípteros Reduviídeos, responsáveis pela transmissão vetorial da doença de Chagas. Atualmente são conhecidas 141 espécies agrupadas em cincos tribos formadas por 18 gêneros ${ }^{1,2,3}$, incluindo a primeira espécie fóssil desse grupo de vetores, recentemente descrita ${ }^{4}$.

Rhodnius brethesi ocorre no Estado do Amazonas, Brasil, é conhecida pela população local como "piolho da piaçaba" e é encontrada no ambiente silvestre em

\author{
Correspondência / Correspondence / Correspondencia: \\ Dayse da Silva Rocha \\ Instituto Oswaldo Cruz \\ Avenida Brasil, $n^{\circ} 4365$ \\ CEP: 21040-900 Rio de Janeiro-Rio de Janeiro-Brasil \\ Tel: +55 (21) 2598-4542 \\ E-mail:dayseroch@gmail.com
}

palmeiras (Leopoldina piassaba). Ao norte do Rio Negro tem ocorrido a transmissão de Trypanosoma cruzi às famílias que trabalham em piaçabais no Município de Barcelos, na extração das fibras dessa palmeira, no ecótopo natural do R. brethesi, ${ }^{5,6,7,8}$.

Na maioria dos países da Bacia Amazônica, tem sido relatada a infecção por $T$. cruzi em humanos, reservatórios e vetores, além de casos autóctones da doença de Chagas. Ao longo das últimas duas décadas tem crescido o interesse pela doença de Chagas na Região Amazônica. Essa região apresenta características enzoóticas sem domiciliação dos vetores e nela se observa a dispersão de espécies nativas, principalmente pertencentes ao gênero Rhodnius. Essa situação poderá sofrer alterações, caso continuem ocorrendo degradações no ambiente silvestre, aliadas às migrações de populações oriundas de outras regiões.

A dispersão passiva dos triatomíneos depende dos hábitos e comportamento dos seus hospedeiros. Diversos autores já relataram o transporte involuntário destes 
insetos em roupas, utensílios e veículos ${ }^{9}$. As penas de aves também servem como excelente meio de dispersão de ovos e até mesmo de ninfas ${ }^{10,11,12,13}$. A dispersão ativa pode ocorrer por meio de deslocamentos de ninfas e adultos ou principalmente por meio do voo dos adultos.

Recentemente, Abrahan et $a^{14}$ avaliaram a capacidade de dispersão ativa (por meio do voo e deslocamento terrestre) e passiva (por meio de galinhas) de Triatoma infestans na Argentina. Os autores apresentaram pela primeira vez dados referentes à recaptura de fêmeas que se deslocaram andando e sugerem que são poucas as possibilidades de galinhas atuarem como portadores passivos de triatomíneos. Do ponto de vista epidemiológico, a dispersão tem grande importância na disseminação da doença de Chagas, principalmente em áreas tratadas que podem sofrer posterior recolonização.

O início da atividade de voo dos triatomíneos pode ser induzido por fatores ambientais, como a elevação da temperatura, ou por necessidades nutricionais. Experimentos de laboratório e campo desenvolvidos por Lehane et $a{ }^{15}$ demonstraram que o início da atividade de voo sofre influência primária do estado nutricional dos insetos e da elevação da temperatura ambiental. Entretanto, Soares e Santoro ${ }^{16}$ verificaram que algumas espécies não iniciam o voo sob nenhuma condição. Durante a ecdise imaginal dos triatomíneos, o desenvolvimento da capacidade de voo parece resultar de alterações morfológicas e adaptações da musculatura envolvida diretamente nesta atividade.

A partir de observações realizadas em laboratório, avaliamos a capacidade de voo de R. brethesi, correlacionando esta atividade a fatores como quantidade de sangue ingerido, gênero e período de jejum.

\section{MATERIAIS E MÉTODOS}

Foram utilizados espécimes de quinto estádio de $R$. Brethesi oriundos de uma colônia aclimatada em laboratório, formada a partir de insetos capturados em palmeiras de piaçaba no rio Aracá, afluente do rio Negro (Estado do Amazonas, Brasil), em 2004. Os espécimes selecionados foram completamente ingurgitados, após um período de $4 \mathrm{~h}$ de alimentação, para a obtenção de adultos. Após a muda imaginal, os insetos (27 machos e 38 fêmeas) foram pesados antes e após a alimentação, para verificação da quantidade de sangue ingerida, e marcados individualmente com tinta guache no pronoto e escutelo, utilizando-se o código de cores baseado no proposto por MacCord et $a^{17}$. A seguir, foram colocados no interior de um equipamento idealizado por Galvão et $a^{18}$ para observação do voo de triatomíneos. Os insetos foram observados diariamente até morrerem, sendo registrados os voos dos espécimes voadores. Todo o experimento foi realizado em temperatura média ambiente de $26+\backslash-5^{\circ} \mathrm{C}$ de temperatura e $70+\backslash-5 \%$ de umidade relativa (UR). Insetos sem asas foram utilizados como grupo controle. Os insetos foram alimentados em pombos (Columba livia), devidamente imobilizados e anestesiados conforme protocolo número L-081-08, aprovado pela Comissão de Ética no Uso de Animais (CEUA) da Fundação Oswaldo Cruz (FIOCRUZ). Os resultados foram analisados estatisticamente por meio do teste $T$ não pareado, considerando SDs iguais (unpaired t test, population with equal SDs) no software GraphPad Instat 3.06.

\section{RESULTADOS}

período médio de sobrevida na fase adulta foi de 17,8 e 22,3 dias para machos e fêmeas, respectivamente. Esse resultado foi analisado por meio do teste $T$ não pareado, apresentando diferença significativa (Tabela 1).

Tabela 1 - Atividade de voo de Rhodnius brethesi Matta, 1919 em laboratório

\begin{tabular}{|c|c|c|c|c|c|}
\hline & Min & Max & Média & $S$ & $S^{2}$ \\
\hline \multicolumn{6}{|l|}{ Período de vida (em dias ) } \\
\hline$(\mathrm{m})$ & 7 & 29 & 17,8 & 35 & 5,81 \\
\hline$(f)$ & 7 & 42 & 22,3 & 63,9 & 7,89 \\
\hline \multicolumn{6}{|l|}{$N^{\circ}$ de voos } \\
\hline (m) & 1 & 6 & 3,7 & 2,684 & 1,600 \\
\hline (f) & 1 & 7 & 4 & 4,032 & 1,975 \\
\hline \multicolumn{6}{|l|}{ Peso inicial após ecdise (mg) } \\
\hline (m) & 0,0729 & 0,1477 & 0,1154 & 0,0004 & 0,0206 \\
\hline$(f)$ & 0,0849 & 0,2079 & 0,1299 & 0,0006 & 0,0255 \\
\hline \multicolumn{6}{|l|}{ Peso final após a morte (mg) } \\
\hline (m) & 0,0326 & 0,0970 & 0,0576 & 0,0002 & 0,0155 \\
\hline$(f)$ & 0,0304 & 0,1069 & 0,0570 & 0,0002 & 0,0143 \\
\hline \multicolumn{6}{|l|}{ Início de voo após repasto } \\
\hline (dias) $(\mathrm{m})$ & 1 & 19 & 11,04 & 17,95 & 4,139 \\
\hline (f) & 2 & 30 & 15,32 & 54,49 & 7,261 \\
\hline \multicolumn{6}{|l|}{ Perda total de peso (mg) } \\
\hline (m) & 0,0301 & 0,0806 & 0,0571 & 0,0001 & 0,0134 \\
\hline$(f)$ & 0,0301 & 0,1589 & 0,0729 & 0,0005 & 0,0240 \\
\hline \multicolumn{6}{|l|}{$\%$ de perda de peso ( $1^{\circ}$ repasto $)$} \\
\hline (m) & 15,2 & 52,2 & 34,14 & 101,08 & 9,811 \\
\hline$(f)$ & 11,5 & 54 & 34,22 & 135,72 & 11,46 \\
\hline
\end{tabular}

$(m)=$ machos $\quad(f)=$ fêmeas 
Não houve diferença significativa entre o número de voos realizados por machos e fêmeas, apesar da diferença significativa encontrada no peso inicial dos insetos, que foi maior nas fêmeas.

Foram observadas diferenças significativas entre a quantidade de sangue ingerido por machos e fêmeas. As fêmeas ingeriram quantidades maiores que os machos e também apresentaram uma perda total de peso durante o período de sobrevida significativamente maior $(P=0,0035)$.

Para o início da atividade de voo, observou-se que a maioria dos insetos necessitou, em média, de 13 dias após a muda imaginal para começar a voar. Verificou-se diferença significativa entre machos e fêmeas, sendo estas as que necessitaram de mais tempo para iniciar o voo (15 dias).

Houve diferenças significativas para tempo de voo, peso inicial e tempo para primeiro voo. O tempo de voo para as fêmeas foi 3,58\% maior que o dos machos; o peso inicial das fêmeas foi $11,53 \%$ maior que o dos machos; e o tempo para primeiro voo foi 4,7\% maior para as fêmeas que para os machos. As fêmeas que voaram mais estavam inicialmente mais pesadas e voaram mais precocemente que os machos.

Os resultados obtidos nas análises comparativas entre número de voos $\mathrm{x}$ perda de peso inicial $\mathrm{x}$ tempo para primeiro voo; \% perda de peso, primeiro voo $x$ tempo para primeirovoo; e tempo de voo x peso médio, estão relacionados nas figuras 1, 2, 3, 4 e tabela 2 .

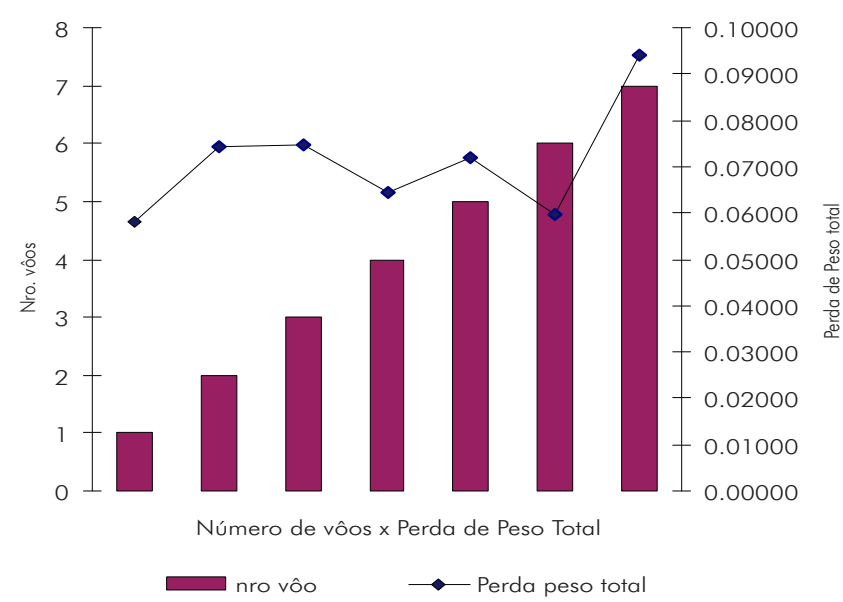

Figura 1 - Número de voos x perda de peso total de Rhodnius brethesi Matta, 1919 em laboratório

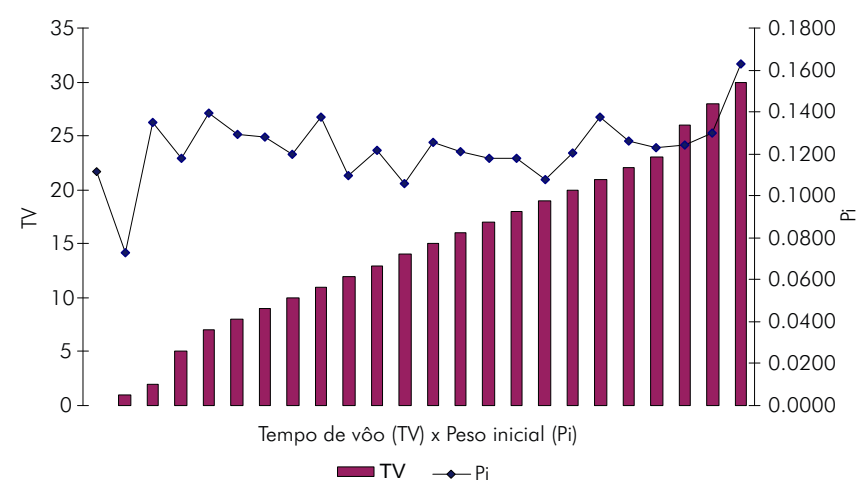

Figura 2 - Tempo de voo x peso inicial de Rhodnius brethesi Matta, 1919 em laboratório

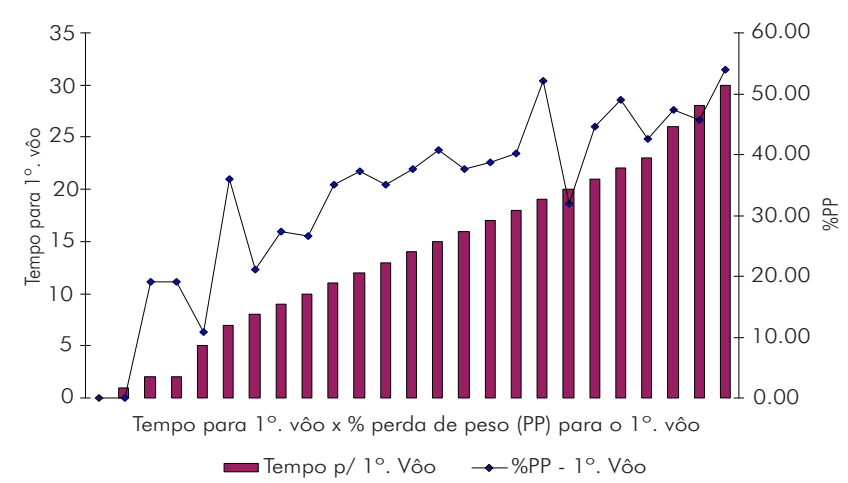

Figura 3 - Tempo para o primeiro voo x perda de peso para o primeiro voo de Rhodnius brethesi Matta, 1919 em laboratório

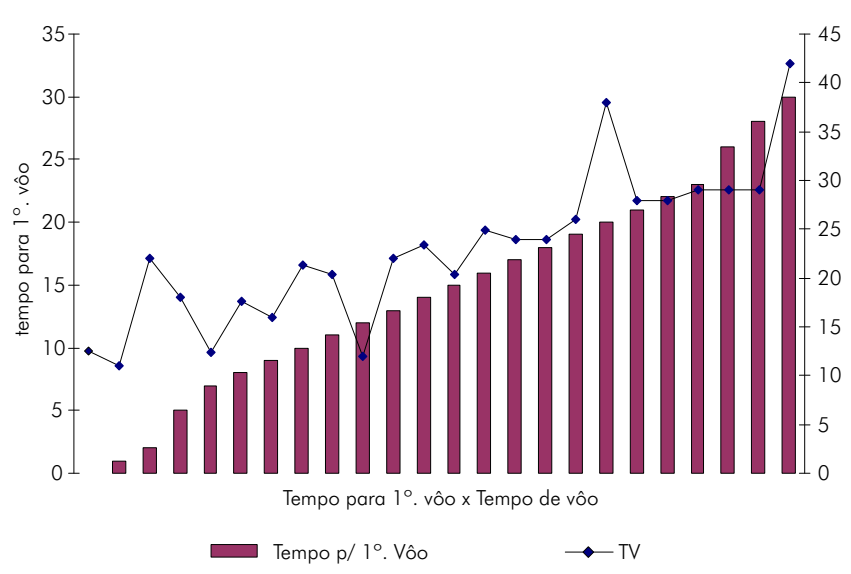

Figura 4 - Tempo para o primeiro voo (em dias) x tempo de voo (em dias) de Rhodnius brethesi Matta, 1919 em laboratório

Tabela 2 - Correlação linear de Person

\begin{tabular}{lcccc}
\hline & $\begin{array}{c}\text { Tempo } \mathrm{p}^{\circ} \\
\text { Voo } \mathrm{TV}\end{array}$ & $\begin{array}{c}\text { Tempo } \mathrm{p} / 1^{\circ} . \\
\text { Voo } \times \% \mathrm{PP} \\
1^{\circ} . \text { Voo }\end{array}$ & TV $\times$ Pi & $\begin{array}{c}\text { N. voo } \times \text { Perda } \\
\text { de Peso Total }\end{array}$ \\
\hline Coef. De Pearson $(r)$ & 0.8373 & 0.8802 & 0.4133 & 0.4775 \\
Valor de P & $\begin{array}{c}<.0001 \\
\text { Ext. sig }\end{array}$ & $\begin{array}{c}<0.0001 \\
\text { Ext. sig. }\end{array}$ & $\begin{array}{c}0.0447 \\
\text { Pouco sig }\end{array}$ & $\begin{array}{c}0.2280 \\
\text { NS }\end{array}$ \\
\hline
\end{tabular}


coeficiente de Pearson ( $r$ ) pode variar de $+1 a-1$ e quanto mais próximos estiverem os resultados desses valores, mais forte será a associação das variáveis.

Os indivíduos que levaram mais tempo para realizar o primeiro voo também realizaram mais voos. Aqueles indivíduos que demoraram mais para realizar o primeiro voo tiveram maior percentual de perda de peso. As variáveis tempo para o primeiro voo x tempo de vida, e tempo para o primeiro voo x percentual de peso perdido para realização do primeiro voo estão fortemente associadas.

Os indivíduos que voaram mais tinham maior peso inicial, sendo essa diferença pouco significativa ( $P=$ 0,4133 ) em relação aos outros indivíduos. Aqueles indivíduos que voaram mais tiveram maior perda de peso total, porém esta diferença não é estatisticamente significativa $(P=0.2280)$.

\section{DISCUSSÃO}

A pequena oferta de hospedeiros em seu ecótopo natural pode ter provocado adaptações no comportamento alimentar de $R$. brethesi, que já foi observado alimentando-se durante o dia em coletores de piaçaba. Rocha et al ${ }^{19}$ observaram o ciclo biológico desta espécie em laboratório e verificaram um excelente aproveitamento do repasto sanguíneo, sendo necessário apenas um repasto para ocorrência de ecdises até o quinto estádio Essa alimentação rápida e eficiente facilitaria a realização de alimentações diurnas utilizando o homem como hospedeiro. Matta ${ }^{20}$ relatou pela primeira vez, em 1922, a agressividade de R. brethesi, que picava por todo - corpo de piaçabeiros, causando inflamações secundárias na pele das pessoas picadas.

O período médio de sobrevida em dias (17,8 machos e 22,3 fêmeas) foi muito inferior ao observado por Mascarenhas $^{21,22}$, de 51,5 para machos e 57,5 para fêmeas, mantidos sob condições próximas às utilizadas no presente trabalho $\left(26^{\circ} \mathrm{C}\right.$ e $75 \%$ UR). Silva e Silva ${ }^{23}$, apesar de terem utilizado temperaturas superiores $\left(30^{\circ} \mathrm{C}\right.$ e $70+\backslash$ $5 \%$ de UR), observaram um período de sobrevida também superior, de 45,2 para machos e 38,9 para fêmeas. Estes resultados sugerem que o curto período registrado neste estudo se deveu à restrição nutricional a que foram submetidos os insetos e ao desgaste provocado pelas tentativas de voo.

Ao serem relacionados o início da atividade de voo e o repasto, foram observados espécimes que voaram no mesmo dia da alimentação. Esse resultado foi inesperado, uma vez que diversos autores atribuem o início da atividade ao baixo estado nutricional e ao aumento médio de temperatura ${ }^{15,24}$. As variáveis tempo para o primeiro voo $x$ tempo de vida e tempo para o primeiro voo $x$ percentual de peso perdido para realização do primeiro voo estão fortemente associadas.

Observamos que 18\% dos insetos não voaram sob nenhuma condição, o que sugere a existência de características intraespecíficas que também influenciam o comportamento de voo dos triatomíneos, conforme observado por Soares e Santoro ${ }^{16}$. Diversos trabalhos têm demonstrado que cada espécie possui um perfil característico e sua caracterização pode ajudar na identificação das espécies que possuem maior capacidade de dispersão ativa; muitos autores têm relacionado a ausência ou diminuição de desenvolvimento dos músculos alares à falta de iniciativa de voo ${ }^{24,25}$.

Observamos um maior número de voos entre as fêmeas, o que também foi observado por Gurevitz et a ${ }^{25}$ para Triatoma infestans, nos quais a ausência de músculos relacionados ao voo foi 2,4 vezes mais frequente nos machos do que em fêmeas.

Rocha et al ${ }^{19}$ observaram a ocorrência de sete repastos sanguíneos, em média, na fase adulta para R. brethesi, o que corresponde a aproximadamente $50 \%$ das tentativas de voo desta espécie. Por meio dos percentuais de perda de peso, observamos que nenhum espécime voou antes de uma perda mínima de $11,5 \%$ de seu peso após o repasto, sendo que a perda média de peso necessária para atividade de voo em laboratório foi de 34\%. Rhodnius brethesi, apresentou total de 81,53\% de insetos voadores.

○ conhecimento das espécies de triatomíneos utilizam o mecanismo de dispersão ativa pelo voo pode ser útil para os programas de vigilância entomológica na Região Amazônica. Durante reunião internacional sobre a implementação da iniciativa intergovernamental de vigilância e prevenção da doença de Chagas, na Amazônia foram relatadas situações particulares de transmissão (entre elas a interferência da população que trabalha na extração das fibras da piaçaba), no ciclo de transmissão silvestre do T. cruzi em seu ecótopo natural. $\bigcirc$ aumento do desmatamento, a construção de casas cada vez mais próximas às florestas, onde são encontradas muitas espécies de palmeiras, a presença de vetores, reservatórios domésticos e silvestres e a iluminação noturna de domicílios, que servem de atração para os insetos, elevam o risco de contato dessa população com as espécies de triatomíneos da Região Amazônica.

\section{CONCLUSÃO}

Os insetos apresentaram uma boa atividade de voo em laboratório, tal como na natureza, conforme descrito por outros autores, que apresentaram relatos de catadores de piaçaba sendo atacados por esta espécie no ambiente silvestre ${ }^{5,6,7}$.

Por meio dos experimentos em laboratórios, conclui-se que esta espécie apresentou uma tendência a dispersar entre 11 e 15 dias após seu último repasto sanguíneo e que, nesse período, sua perda de peso correspondeu a $34 \%$ de seu peso inicial. Conclui-se ainda que R. brethesi necessita de uma perda mínima de $11 \%$ de seu peso para que seja iniciada a atividade de voo para suprir as suas necessidades nutricionais. $\bigcirc$ desmatamento crescente, com consequente eliminação das fontes alimentares silvestres, leva o inseto ao jejum, induzindo, em uma ou duas semanas, sua dispersão pelo voo, que pode ser orientado pela presença de luz em habitações cada vez mais próximas às áreas desmatadas. 


\title{
Laboratory analysis of the flight of Rhodnius brethesi Matta, 1919, potential wild vector of Trypanosoma cruzi in the Brazilian Amazon. (Hemiptera:Reduviidae:Triatominae)
}

\begin{abstract}
The flight ability of Rhodnius brethesi was evaluated through laboratory observations, and it was correlated with the quantity of blood ingested, gender and fasting period. A total of 65 insects were analyzed: 27 males and 38 females; their mean survival period was 17.8 and 22.3 days, respectively. Most of the insects started flying after 13 days.
\end{abstract}

Keywords: Rhodnius; Chagas Disease; Triatominae; Insect Vectors; Behavior; Animal; Flight, Animal.

\section{Evaluación en laboratorio de la actividad de vuelo de Rhodnius brethesi Matta, 1919, potencial vector silvestre del Trypanosoma cruzi en la Amazonía Brasileña. (Hemiptera:Reduviidae:Triatominae)}

\section{RESUMEN}

A partir de observaciones realizadas en laboratorio, fue evaluada la capacidad de vuelo de Rhodnius brethesi, correlacionando esta actividad a factores como cantidad de sangre ingerida, género y período de ayuno. Fueron observados 27 machos y 38 hembras. El período medio de supervivencia fue de 17,8 y 22,3 días para machos y hembras, respectivamente. La mayoría de los insectos necesitó un promedio de 13 días para iniciar el vuelo.

Palabras clave: Rhodnius; Enfermedad de Chagas; Triatominae; Insectos Vectores; Comportamiento Animal; Vuelo Animal.

\section{REFERÊNCIAS}

1 Galvão C, Carcavallo RU, Rocha DS, Jurberg J. A checklist of the current valid species of the subfamily Triatominae Jeannel, 1919 (Hemíptera, Reduviidae) and their geographical distribution, with nomenclatural and taxonomic notes. Zootaxa. 2003;202:1-36.

2 Galvão C, Angulo VM. Belminus corredori, a new species of Bolboderini (Hemiptera: Reduviidae: Triatominae) from Santander, Colombia. Zootaxa. $2006 ; 1241: 61-8$.

3 Schofield CJ, Galvão C. Classification, evolution, and species groups within the Triatominae. Acta Trop. 2009 May-Jun;1 10(2-3):88-100.

4 Poinar Jr G. Triatoma dominicana sp.n. (Hemiptera: Reduviidae: Triatominae), and Trypanosoma antiquus sp.n. (Stercoraria: Trypanosomatidae), the first fossil evidence of a triatomine-trypanosomatid vector association. Vector Borne Zoonotic Dis. 2005;5(1):72-81.

5 Coura JR, Arboleda MN, Willcox HPF. Doença de Chagas na Amazônia brasileira. Rev Soc Bras Med Trop. 1993;26 Suppl 2:15-17.

6 Coura JR, Barreu TV, Arboleda MN. Ataque de populações humanas por triatomíneos silvestres no Amazonas: uma nova forma de transmissão da infecção chagásica? Soc Bras Med Trop. 1994 outdez;27(4):251-4.

7 Coura JR, Arboleda MN, Willcox HPF. Chagas disease in the Brazilian Amazon. II-a serological survey. Rev Med Trop Sao Paulo. 1995 MarApr,37(2): 103-7.
8 Coura JR, Junqueira ACV, Boia MN, Fernandes $\mathrm{O}$. Chagas disease: from bush to hus and houses. Is it the case of the Brazilian Amazon? Mem Inst Oswaldo Cruz. 1999;94(Suppl 1):378-84.

9 Miles MA. Human behaviour and the propagation of Chagas disease. Trans R Soc Trop Med Hyg. 1976;70(5-6):521-2.

10 Gamboa CJ. Dispersion de Rhodnius prolixus em Venezuela. Bol Dir Malariol San Amb. 1962;3:26272.

11 Forattini OP, Ferreira AO, Rocha-Silva EO, Rabello EX, Santos JL. Aspectos ecológicos da tripanosomose americana. II - Distribuição e dispersão local de triatomíneos em ecótopos naturais e artificiais. Rev Saude Publica. 1971 dez;5(2):163-91.

12 Forattini OP, Rocha-Silva EO, Ferreira AO, Rabello EX, Patolli DGB. Aspectos ecológicos da tripanosomose americana. III - Dispersão local de triatomíneos, com especial referência ao Triatoma sórdida. Rev Saude Publica. 1971;5:193-205.

13 Ribeiro Jr G, Silva-Santos CG, Noireau F, Dias-Lima A. Potencial de dispersão de algumas espécies de Triatomíneos (Hemiptera: Reduviidae) por aves migratórias. Ser Ci Biol. 2006;6(4):324-8.

14 Abrahan LB, Gorla DE, Catalá SS. Dispersal of Triatoma infestans and other. Triatominae species in the arid Chaco of Argentina - Flying, walking or passive carriage? The importance of walking females. Mem Inst Oswaldo Cruz. 2011 Mar; 106(2):232-9.

15 Lehane MJ, Mcewan PK, Whitaker CJ, Schofield CJ. The role of temperature and nutritional status in flight initiation by Triatoma infestans. Acta trop.1992 Sep;52(1):27-38. 
16 Soares RPP, Santoro MM. a-glycerophosphate dehydrogenase activity in flight muscles of Triatominae bugs Panstrongylus megistus and Triatoma sordida. Mem Inst Oswaldo Cruz. 2000 Sep-Oct;95(5):707-9.

17 Mac CJR, Jurberg P, Lima MM. Marcação individual de triatomíneos para estudos comportamentais e ecológicos. Mem Inst Oswaldo Cruz. 1983 outdez;78:473-6.

18 Galvão C, Rocha DS, Jurberg J, Carcavallo RU. Início da atividade de vôo em Triatoma infestans (Klug, 1834) e T. melanosoma Martínez, Olmedo \& Carcavallo, 1987 (hemiptera, Reduviidae). Mem Inst Oswaldo Cruz. 2001 jan;96(1):137-40.

19 Rocha DS, Magalhães CS, Cunha V, Jurberg J, Galvão C. Ciclo biológico em laboratório de Rhodnius brethesi Matta, 1919 (Hemíptera, Reduviidae, Triatominae), potencial vetor silvestre da Doença de Chagas na Amazônia. Mem Inst Oswaldo Cruz. 2004 out, 99(6):591-5.

20 Matta A. Sobre o gênero Rhodnius do Amazonas. Amazonas Med. 1922; 5:161-2.

21 Mascarenhas BM. Triatomíneos da Amazônia. Sobre o ciclo evolutivo de Rhodnius brethesi Matta, 1919 (Hemíptera, Reduviidae, Triatominae). Bol Mus Par Emilio Goeldi Série Zol. 1990;6:191-202.
22 Mascarenhas BM. Triatomíneos da Amazônia: sobre o habitat e algumas considerações comportamentais de Rhodnius brethesi Matta, 1919 (Hemiptera, Reduviidae: Triatominae) na região do médio Rio Negro, Amazonas. Bol Mus Par Emilio Goeldi Série Zol. $1991 ; 7(2): 107-16$.

23 Silva IG, Silva HHG. Influência da temperatura na biologia de triatomíneos. X. Triatoma vitticeps Stal, 1859 (Hemiptera, Reduviidae). Rev Goiana Med. 1988; jan-jun.34(1/2):39-45.

24 Gurevitz JM, Ceballos LA, Kitron U, Gurtler RE. Flight initiation of Triatoma infestans (Hemiptera: Reduviidae) under natural climatic conditions. J Med Entomol. 2006 Mar;43(2): 143-50.

25 Gurevitz JM, Kitron U, Gurtler RE. Flight muscle dimorphism and heterogeneity in flight initiation of field-collected Triatoma infestans (Hemiptera: Reduviidae) . J Med Entomol. 2007; Mar;44(2):18691. 